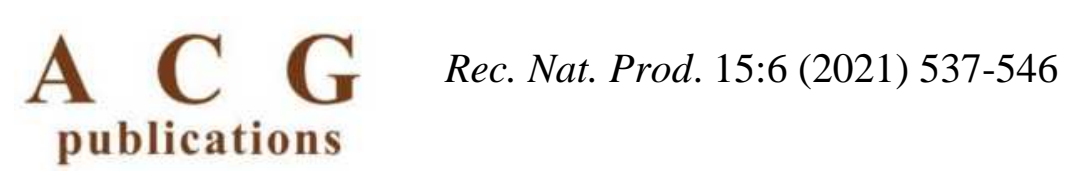

records of natural products

\title{
Saponins from Twenty-Two Cephalaria Species
}

\section{Nazli Boke Sarikahya $\oplus^{1 *}$, Ahmet C. Goren ${ }^{2,3^{*}}$, Gaye Sumer Okkali $\oplus^{1}$ and Suheyla Kirmizigul 1}

\author{
${ }^{1}$ Department of Chemistry, Faculty of Science, Ege University, 35100 Bornova-Izmir, Türkiye \\ ${ }^{2}$ Department of Analytical Chemistry, Faculty of Pharmacy, Bezmialem Vakif University, 34093 \\ Istanbul, Türkiye \\ ${ }^{3}$ Drug Application and Research Center (DARC), Bezmialem Vakif University, 34093 Istanbul, Türkiye
}

(Received February 15, 2021; Revised April 15, 2021; Accepted April 18, 2021)

\begin{abstract}
Saponins in the Cephalaria species have a broad spectrum of biological properties and application potentials. This research aimed to develop a novel method to tentatively identify and quantify triterpenes and saponins in 22 Cephalaria species by LC-HR/MS method. In this study, we provided a broad screening of important biologically active triterpenes and saponins with high separation ability, high sensitivity and high selectivity. This is the first report to prove that Cephalaria species can be a good source of tormentic acid which has many biological activities in literature. The main triterpene compound, tormentic acid was presented at the level between 74.02 $\mathrm{mg} / \mathrm{kg}$ and $75702.0 \mathrm{mg} / \mathrm{kg}$ in $n$-butanol extract of Cephalaria species. The source of some biologically active saponins such as davisianoside B, aristatoside C, elmalienoside A, balansoide B and scoposide B were also determined with this study, for the first time. Therefore, due to the different activities and usages of the identified compounds, these findings may find application in a number of industries such as nutraceutical, pharmaceutical, medical and cosmetic industries.
\end{abstract}

Keywords: Cephalaria; Caprifoliaceae; triterpene; saponin; validation; LC-HR/MS. 2021 ACG Publications. All rights reserved.

\section{Introduction}

Saponins are glycosides, containing steroid or triterpenic aglycone and one or more carbohydrate side chains, and have become increasingly important in human health researches, in recent years. Plants containing saponins are known to have many biological effects, especially immunostimulating, antiinflammatory, antimicrobial, anticancer, hypocholesterolemic and hemolytic effects [1-4]. Due to these various chemical and biological properties, they have commercial applications in many sectors such as cosmetics, cleaning, food, agriculture and pharmacy. In addition, their natural non-ionic surfactant properties cause them to find different usages in many other industries [1]. Researches and evidence on their biological activities have brought interest in saponins in recent years. It is known that these

\footnotetext{
* Corresponding authors: E-mail: nazli.sarikahya@ege.edu.tr; (N.Boke-Sarikahya) phone: +90232 3112366. Fax: +90232 3888264. ahmetcgoren@yahoo.com (A.C. Goren)
} 


\section{Saponins from Cephalaria species}

compounds are generally used as vaccine adjuvant, immune system regulators and anti-microbial agents in pharmaceutical applications [5-7]. Cephalaria genus is a class of plants that belongs to the Caprifoliaceae family and is related to many different biological activities in the world with its extremely rich chemical content [8-10]. Many different activities such as antimicrobial, moluscicidal, antifungal, antioxidant and cytotoxic have also enabled the plant to find use in many fields from medicine to cosmetics [11,12]. Cephalaria genus, which has 94 species in the world has 40 species in Turkey, 24 of which are endemic. Most of the species are mainly located in the Mediterranean region and the Middle East $[13,14]$. Oleanane-type triterpene saponins were determined as one of the major bioactive ingredients in Cephalaria species. So far, more than new 50 saponin compounds have been isolated and identified from the $n$-butanol extracts of different Cephalaria species from Turkey [8, 15-21]. These saponins exhibit many important biological properties such as cytotoxicity, hemolytic and immunomodulatory effects [16].

However, up to now, no chromatographic methods have been developed to provide full fingerprinting and quality assurance for these saponins of Cephalaria species. Thus, in this study LCHR/MS method was developed to profile bioactive saponin contents of $n$-butanol extracts of 22 different Cephalaria species, for the first time. Nowadays LC-HR/MS is one of the most widely-used bioanalytical methods, which has the advantages of high separation ability, high sensitivity and high selectivity for large molecule quantification in biological samples. The detected major compounds such as tormentic acid, dipsacus saponin A and $\alpha$-hederin have potential interest in the development of new clinically relevant drugs and industrial areas [22-24].

\section{Materials and Methods}

\subsection{Plant Materials}

All studied Cephalaria species, which were harvested in Anatolia, during the vegetative season of 2013 by R.S. Gokturk. Identifications of the plants were established by H. Sumbul and R. S. Gokturk from Akdeniz University. Voucher specimens have been deposited at the Herbarium of the Biology Department of Akdeniz University. All details such as names, voucher specimen numbers, localities and heights about the plant materials (see Sup. Info. Figure S1, Table S1) have been given in our previous study [25] except three species. C. gazipashaensis Sumbul (RSG6099), C. hirsuta Stapf (RSG7676) and C. cilicica Boiss \& Kotschy (RSG 6076) were collected from Gazipasa, Maha: Antalya 1500 m, CatErzurum $2100 \mathrm{~m}$ and Finike-Elmali: Antalya, 1200 m, respectively.

\subsection{Chemicals}

Following compounds which were isolated and purified in our previous studies were used as standards for method validation in LC-HRMS analysis. Gazipashoside B (>99.9\%) [19], aristatoside A (>98.8\%), aristatoside C $(>93.7 \%)$ [17], cephoside B (>99.1\%) [26], davisianoside A $(>97.5 \%)$, davisianoside B (>97.6\%) [27], elmalienoside A (>99.6\%), elmalienoside C (>99.9\%) [18], balansoid A (>99.3\%), balansoid B (>98.5\%), balansoid C (>99.8\%) [15], dipsacoside B (>99.2\%) [28], tchihatchewoside A (>99.1\%), tchihatchewoside B (>99.7\%) [29], scoposide B (>99.9\%) [8], scoposide G (>99.6\%) [30], saponin I (>99.7\%) [31], saponin II (>99.9\%) [32], sumbulianoside A (>99.5\%) [33], isacoside (>96.5\%) [34], akebia saponin F (>97.5\%) [35], sapindoside C (>99.8\%) [36], lycicoside II (>98.7\%) [37], macranthoside A (>98.6\%) [38], collinsonidin (>98.6\%) [39], dipsacus saponin A (>99.1\%) [40], $\alpha$-hederin (>99.8\%) [41], anemoclemoside A (>99.2\%) [42], oleanoic acid (>95\%) [17], pomolic acid (>97\%), tormentic acid (>97\%) [43], ursolic acid (isolated from Satureja species, 97\%).

\subsection{Preparation of Plant Extracts and Sample Preparation for LC-HRMS}

After all plants were collected, the aerial parts of the plants were dried and ground. Grinded samples (each approx. $100 \mathrm{~g}$ ) were extracted three times with methanol (each $10 \mathrm{~mL}$ ) by a laboratory homogenizer (Silverson, L5M-A, USA) for $3 \mathrm{~h}$ at room temperature. The extracts were concentrated under reduced pressure till dryness at $40{ }^{\circ} \mathrm{C}$. And then, $\mathrm{MeOH}$ residues were extracted three times with $n$ - $\mathrm{BuOH}$ $: \mathrm{H}_{2} \mathrm{O}(1: 1)$ solvent system (each $5 \mathrm{~mL}$ ). After the separation and evaporation of $n-\mathrm{BuOH}$ and $\mathrm{H}_{2} \mathrm{O}$ 
fractions, the saponin-riched $n$ - $\mathrm{BuOH}$ fractions were concentrated under reduced pressure (extract yields 2.5-3.0\%). The dried $100 \mathrm{mg}$ of the $n$-butanol extracts of Cephalaria species were dissolved in approximately $4 \mathrm{~mL}$ of methanol-water mixture $(60: 40, \mathrm{v} / \mathrm{v})$ in a $5 \mathrm{~mL}$ volumetric flask. The flask was kept in an ultrasonic bath for $20 \mathrm{~min}$. After a clear solution obtained $100 \mu \mathrm{L}$ of dihydrocapsaicin solution (from $100 \mathrm{ppm}$ stock solution) was added as an internal standard and diluted to the volume with mobile phase of the LC-HRMS measurement, and afterwards the mixture was warmed to get a homogenous solution. The final solution was filtered through a $0.45 \mu \mathrm{m}$ Millipore Millex-HV filter and the concentration of final solution $(1 \mathrm{~mL})$ was transferred into a capped auto sampler vial, from which $2 \mu \mathrm{L}$ of sample was injected to LC for each run. The samples in the auto sampler were kept at $15{ }^{\circ} \mathrm{C}$ during the experiment $[17,25,44-46]$.

\subsection{Instruments and Chromatographic Conditions of LC-HRMS}

LC-HRMS measurements were carried out on a Thermo ORBITRAP Q-EXACTIVE (Bremen, Germany) mass spectrometry equipped ESI ion source and with Dionex LC system. Scan range was set to $\mathrm{m} / \mathrm{z}$ 300-1600 amu and other mass paramaters are used as following: gas flow rate:45, aux gas flow rate: 10 , sprey voltage: $3.80 \mathrm{kV}$, capilary temperature: $320^{\circ} \mathrm{C}$, aux gas heater temperature: $320{ }^{\circ} \mathrm{C}$, and Slens RF is 50. A Troyasil C18 column ( 150 x $3 \mathrm{~mm}$ i.d., $3 \mu \mathrm{m}$ particle size, Istanbul, Turkey) was for separation of compounds. The mobile phases $\mathrm{A}$ and $\mathrm{B}$ were composed of $1 \%$ formic acid-water and $1 \%$ formic acid-methanol, respectively. The gradient program of which was started $50 \%$ A and $50 \% \mathrm{~B}, 0.01$ $1 \mathrm{~min}, 75 \% \mathrm{~B}, 1.01-3.00 \mathrm{~min} 85 \% \mathrm{~B}, 3.01$ and $15.0090 \% \mathrm{~B}$, and finally $15.01-25 \mathrm{~min} 50 \% \mathrm{~A}$ and $50 \%$ B. The flow rate of the mobile phase was $0.35 \mathrm{~mL} / \mathrm{min}$, and the column temperature was set to $22^{\circ} \mathrm{C}$. Environmental conditions were set as temperature $22.0 \pm 5.0^{\circ} \mathrm{C}$ and relative humidity $(50 \pm 15) \%$ rh [4448].

\subsection{Method Validation}

The validation parameters consisted of selectivity, linearity, repeatability, recovery, limit of detection (LOD) and limit of quantification (LOQ) experiments. The linearity for each compound for the reported method was determined by analysing standard solutions. Repeatability, and intermediate precision of the validated method used and screening measurements were done by using the extract of $C$. tchihatchewii Boiss. Recovery of method was conducted to determine the accuracy of the method for the determination of the amount of substances in plant extracts for the target compounds and the method was checked at around concentration of $0.5 \mathrm{mg} / \mathrm{L}$ and $5 \mathrm{mg} / \mathrm{L}$. Simply standard compounds are spiked the diluted extract to reach the desired concentrations, dissolved in methanol in a $5 \mathrm{~mL}$ volumetric flask. Unspiked diluted plant extracts were also analyzed to determine the target compounds concentrations in the blank sample. The recoveries of the target compounds ranged from 92.90 to $109.40 \%$ (Table 1). Mean relative standard deviations (RSD) were found to be $0.1-10 \%$ for all and that data was added to the uncertainty budget of all reported compounds. The summary of validation and uncertainty data, the correlation coefficients $\left(\mathrm{r}^{2}\right)$, LOD, LOQ, recovery, and linear regression equations of the reported compounds are presented in Table 1, where $\mathrm{y}$ is the peak area and $\mathrm{x}$ is the concentration. Detailed methodology of validation procedure and uncertainty evaluation of the used LC-HRMS method were reported in our previous papers [47-49].

\section{Results and Discussion}

In this study, the detailed triterpene saponin contents of $n$-butanol extracts of 22 Cephalaria species were determined by LC-HRMS, for the first time (Table 2). The twenty-four different saponins (gazipashoside B, aristatosides A and C, cephoside B, davisianosides A-B, elmalienosides A and C, balansoids A-C, dipsacoside B, tchihatchewosides A-B, scoposide B, saponin I, isacoside, akebia saponin F, sapindoside $\mathrm{C}$, lycicoside II, macranthoside A, collinsonidin, dipsacus saponin A, $\alpha$-hederin) and two triterpene aglycones (pomolic acid, tormentic acid), totally twenty-six compounds were detected 


\section{Saponins from Cephalaria species}

simultaneously. Table 2 summarizes the content of each saponin and triterpene in the identified 22 Cephalaria species.

Table 1. Mass parameters and linear regression equation of compounds for 22 Cephalaria species from Turkey.

\begin{tabular}{|c|c|c|c|c|c|c|c|c|}
\hline Compounds & $m / z$ & $\begin{array}{c}\text { Ionization } \\
\text { Mode }\end{array}$ & $\begin{array}{l}\text { Linear } \\
\text { Range* }^{*}\end{array}$ & $\begin{array}{c}\text { Linear Regression } \\
\text { Equation }\end{array}$ & $\begin{array}{l}\text { LOD/ } \\
\text { LOQ }\end{array}$ & $\mathbf{r}^{2}$ & $\begin{array}{c}\text { Rec. } \\
\%\end{array}$ & $\begin{array}{l}\mathbf{U} \\
\%\end{array}$ \\
\hline Gazipashoside B & 1390.6375 & Positive & $5-10$ & $\mathrm{y}=0.00005686 \mathrm{x}-0.00001284$ & $1.66 / 5.0$ & 0.994 & 103.2 & 1.7 \\
\hline Aristatoside A & 1543.7173 & Negative & $0.5-10$ & $\mathrm{y}=0.002781 \mathrm{x}-0.0004899$ & $0.18 / 0.6$ & 0.999 & 101.4 & 4.0 \\
\hline Cephoside B & 1397.6594 & Negative & $0.25-5$ & $y=0.03048 x-0.003199$ & $0.08 / 0.26$ & 0.998 & 102.8 & 11.2 \\
\hline Davisianoside A & 1381.6645 & Negative & $0.5-10$ & $\mathrm{y}=0.007459 \mathrm{x}-0.0003556$ & $0.2 / 0.67$ & 0.999 & 104.8 & 5.9 \\
\hline Elmalienoside C & 1229.5925 & Positive & $0.5-10$ & $y=-0.0003556 x-0.03472$ & $0.29 / 0.96$ & 0.994 & 93.4 & 10.2 \\
\hline Balansoid C & 1376.6582 & Positive & $0.5-10$ & $y=0.008914 x+0.002941$ & $0.28 / 0.92$ & 0.997 & 106.1 & 10.1 \\
\hline Dipsacoside B & 1075.5683 & Positive & $0.5-10$ & $y=0.003923 x-0.0003301$ & $0.15 / 0.5$ & 0.999 & 108.6 & 3.5 \\
\hline Elmalienoside A & 1259.6031 & Positive & $0.5-10$ & $y=0.03443 x+0.000459$ & $0.12 / 0.41$ & 0.999 & 107.8 & 13.3 \\
\hline Tchihatchewoside A & 952.5002 & Positive & $0.5-10$ & $y=0.02322 x-0.001075$ & $0.11 / 0.36$ & 0.999 & 106.4 & 4.3 \\
\hline Saponin II & 1075.568 & Positive & $0.5-10$ & $\mathrm{y}=0.001301 \mathrm{x}+0.0008106$ & $0.48 / 1.61$ & 0.993 & 108.3 & 8.2 \\
\hline Scoposide B & 1351.653 & Negative & $0.1-2$ & $y=0.08182 x+0.0002178$ & $0.02 / 0.08$ & & 98.0 & 0.9 \\
\hline Tchihatchewoside B & 1090.556 & Negative & $0.2-5$ & $y=0.03348 x+-0.002113$ & $0.22 / 0.73$ & 0.996 & 95.5 & 9.8 \\
\hline Saponin I & 935.4974 & Positive & $0.5-10$ & $\mathrm{y}=0.1289 \mathrm{x}-0.006194$ & $0.15 / 0.50$ & 0.999 & 102.2 & 7.9 \\
\hline Sumbulianoside A & 820.4579 & Positive & $0.5-7$ & $y=0.1222 x+0.02024$ & $0.17 / 0.56$ & 0.999 & 100.8 & 4.9 \\
\hline Balansoid B & 1189.601 & Negative & $01-2$ & $y=0.07515 x-0.002736$ & $0.06 / 0.19$ & 0.997 & 99.0 & 19.4 \\
\hline Aristatoside $\mathrm{C}$ & 1175.585 & Negative & $0.5-10$ & $\mathrm{y}=0.01879 \mathrm{x}-0.002058$ & $0.13 / 0.44$ & 0.999 & 92.9 & 4.6 \\
\hline Isacoside & 1353.668 & Positive & $0.5-10$ & $\mathrm{y}=0.001775 \mathrm{x}-0.0005579$ & $0.24 / 0.81$ & 0.999 & 97.9 & 10.0 \\
\hline Scoposide G & 1189.601 & Negative & $0.5-10$ & $y=0.003077 x-0.0003519$ & $0.14 / 0.48$ & 0.999 & 102.3 & 1.0 \\
\hline Akebia Saponin F & 1073.553 & Negative & $0.2-5$ & $\mathrm{y}=0.03514 \mathrm{x}-0.001404$ & $0.03 / 0.1$ & 0.999 & 100.2 & 8.8 \\
\hline Sapindoside C & 1045.557 & Positive & $0.5-10$ & $y=0.004917 x-0.001086$ & $0.2 / 0.67$ & 0.999 & 98.3 & 3.8 \\
\hline Tormentic acid & 511.3394 & Positive & $01-2$ & $y=0.08359 x+0.003066$ & $0.03 / 0.11$ & 0.998 & 101.0 & 3.5 \\
\hline Balansoid A & 1027.548 & Negative & $0.5-10$ & $y=0.02999 x-0.002864$ & $0.19 / 0.62$ & 0.998 & 102.3 & 4.5 \\
\hline Davisianoside B & 1059.573 & Positive & $0.5-10$ & $y=0.00563 x-0.001216$ & $0.12 / 0.40$ & 0.999 & 106.5 & 7.7 \\
\hline Lycicoside II & 1029.562 & Positive & $0.5-10$ & $\mathrm{y}=0.002458 \mathrm{x}-0.0001271$ & $0.07 / 0.24$ & 0.999 & 102.9 & 8.1 \\
\hline Macranthoside A & 935.4974 & Positive & $0.5-10$ & $\mathrm{y}=0.05647 \mathrm{x}-0.004836$ & $0.09 / 0.29$ & 0.999 & 102.7 & 11.1 \\
\hline Collinsonidin & 789.4395 & Positive & $0.5-10$ & $\mathrm{y}=0.04576 \mathrm{x}-0.0008392$ & $0.18 / 0.6$ & 0.998 & 109.4 & 8.0 \\
\hline Dipsacus saponin A & 796.4614 & Negative & $0.5-10$ & $y=0.06377 x-0.01101$ & $0.2 / 0.67$ & 0.998 & 109.4 & 6.7 \\
\hline$\alpha$-hederin & 749.4481 & Negative & $0.5-10$ & $y=0.01466 x-0.003416$ & $0.23 / 0.75$ & 0.997 & 109.1 & 4.6 \\
\hline Pomolic acid & 511.3394 & Positive & $0.5-10$ & $\mathrm{y}=0.005607 \mathrm{x}-0.001334$ & $0.17 / 0.56$ & 0.999 & 100.9 & 1.7 \\
\hline Anemoclemoside A & 603.39024 & Negative & $0.5-10$ & $\mathrm{y}=0.00189 \mathrm{x}-0.0005119$ & $0.37 / 1.25$ & 0.998 & 106.2 & 12.8 \\
\hline
\end{tabular}

*ppm, Rec: Recovery

Among the detected compounds, 3 saponins in C. elmaliensis, 8 saponins in C. elazigensis var. elazigensis, 9 saponins in C. elazigensis var. purpurea, 12 saponins in C. stellipilis, 13 saponins in $C$. speciosa, C. gazipashaensis and C. tuteliana, 14 saponins in C. cilicica and C. scoparia, 15 saponins in C. isaurica and C. hirsuta, 16 saponins in C. tchihatchewii, C. davisiana, C. aristata, C. balansae, C. lycica and $C$. procera, 17 saponins in $C$. sumbuliana, 18 saponins in $C$. taurica, $C$. aytachii and $C$. anatolica, 20 saponins in C. paphlagonica were identified and quantified based on their retention times and MS/MS pattern in comparison with the data of references. While as a triterpene tormentic acid detected in all Cephalaria species, pomolic acid was detected in 8 Cephalaria species (C. tuteliana, C. aytachii, C. davisiana, C. balansae, C. elazigensis var. elazigensis, C. anatolica, C. stellipilis and C. cilicica). 
Boke Sarikahya et.al., Rec.Nat.Prod. 15:6 (2021) 537-546

Table 2. Compounds and their amounts ( $\mathrm{mg} / \mathrm{kg}$ extract) of 22 Cephalaria species from Turkey

\begin{tabular}{|c|c|c|c|c|c|c|c|c|c|c|c|}
\hline \multirow{2}{*}{ Compounds } & \multicolumn{11}{|c|}{ Cephalaria Species } \\
\hline & $\mathbf{1}$ & 2 & 3 & 4 & 5 & 6 & 7 & 8 & 9 & 10 & 11 \\
\hline Gazipashoside B & $<\mathrm{LOD}$ & $<\mathrm{LOD}$ & $<\mathrm{LOD}$ & $<\mathrm{LOD}$ & $<\mathrm{LOD}$ & $<\mathrm{LOD}$ & $<\mathrm{LOD}$ & $<\mathrm{LOD}$ & $<\mathrm{LOD}$ & 13635.8 & $<\mathrm{LOD}$ \\
\hline Aristatoside A & $<\mathrm{LOD}$ & 30.3 & 613.2 & 14.0 & 29.7 & 208.9 & 86.8 & 134.3 & 814.7 & $<\mathrm{LOD}$ & 538.8 \\
\hline Cephoside B & 7.5 & 17.2 & 57.3 & 42.4 & 8.4 & 28.3 & 331.2 & 149.6 & 149.2 & 5588.5 & 82.9 \\
\hline Davisianoside A & 4.8 & 18.2 & 96.8 & 224.7 & 115.7 & 63.8 & 1285.2 & 179.6 & 136.3 & 244.4 & 443.4 \\
\hline Elmalienoside $\mathrm{C}$ & 6.58 & 22.8 & 15.0 & 0.5 & 55.8 & 0.9 & 1.6 & 49.6 & 41.6 & 1907.6 & 925.7 \\
\hline Balansoid C & $<\mathrm{LOD}$ & $<\mathrm{LOD}$ & $<\mathrm{LOD}$ & $<\mathrm{LOD}$ & $<\mathrm{LOD}$ & $<\mathrm{LOD}$ & $<\mathrm{LOD}$ & 264.7 & $<\mathrm{LOD}$ & 14776.4 & $<\mathrm{LOD}$ \\
\hline Dipsacoside B & 777.7 & 79.2 & $<\mathrm{LOD}$ & 2215.8 & 5530.1 & 143.0 & 2608.6 & 346.8 & 743.2 & 6129.0 & 2713.0 \\
\hline Elmalienoside A & 17.4 & 1.8 & 16.0 & 103.5 & 111.0 & 5.5 & 778.4 & 46.0 & 71.6 & 64.7 & 121.4 \\
\hline TchihatchewosideA & 476.2 & 33.5 & $<\mathrm{LOD}$ & 122.2 & 350.5 & 13.3 & 735.0 & 106.2 & $<\mathrm{LOD}$ & 1423.2 & 948.4 \\
\hline Scoposide B & 0.4 & 105.4 & $<\mathrm{LOD}$ & $<\mathrm{LOD}$ & 1.4 & $<\mathrm{LOD}$ & $<\mathrm{LOD}$ & 3.2 & $<\mathrm{LOD}$ & $<\mathrm{LOD}$ & 11.7 \\
\hline TchihatchewosideB & $<\mathrm{LOD}$ & 14.9 & 55.7 & $<\mathrm{LOD}$ & 9.5 & 4.9 & $<\mathrm{LOD}$ & $<\mathrm{LOD}$ & $<\mathrm{LOD}$ & 207.0 & 1991 \\
\hline Saponin I & 11.6 & $<\mathrm{LOD}$ & 15.0 & 41.3 & 97.6 & 5.1 & 102.8 & 66.2 & 45.5 & 476.8 & 18.1 \\
\hline Balansoid B & 2.9 & 208.6 & 9.5 & 1.9 & 18.0 & 2.9 & 7.4 & $<\mathrm{LOD}$ & $<\mathrm{LOD}$ & 131.8 & 54.6 \\
\hline Aristatoside C & 3.4 & 14.1 & 22.3 & $<\mathrm{LOD}$ & $<\mathrm{LOD}$ & 7.8 & 175.4 & $<\mathrm{LOD}$ & 7030.8 & $<\mathrm{LOD}$ & 18.4 \\
\hline Isacoside & $<\mathrm{LOD}$ & $<\mathrm{LOD}$ & 1981.3 & $<\mathrm{LOD}$ & 11.6 & 742.3 & $<\mathrm{LOD}$ & $<\mathrm{LOD}$ & 2084.2 & $<\mathrm{LOD}$ & 223.3 \\
\hline Akebia Saponin F & $<\mathrm{LOD}$ & 181.9 & 35.5 & $<$ LOD & $<\mathrm{LOD}$ & 16.9 & 110.8 & 30.4 & 3775.5 & $<\mathrm{LOD}$ & 22.5 \\
\hline Sapindoside C & 7.1 & $<\mathrm{LOD}$ & $<\mathrm{LOD}$ & $<\mathrm{LOD}$ & $<\mathrm{LOD}$ & $<\mathrm{LOD}$ & 325.6 & $<\mathrm{LOD}$ & 19784.5 & $<\mathrm{LOD}$ & $<\mathrm{LOD}$ \\
\hline Tormentic acid & 117.7 & 3342.1 & 4827.5 & 764.5 & 323.7 & 2165.9 & 11143.6 & 13658.5 & 11003.7 & 3004.9 & 4765.2 \\
\hline Balansoid A & 7.6 & $<\mathrm{LOD}$ & 16.2 & 2.7 & 3.9 & 6.5 & 20.0 & 41.9 & 81.6 & 1483.5 & 76.2 \\
\hline Davisianoside B & $<\mathrm{LOD}$ & $<\mathrm{LOD}$ & $<\mathrm{LOD}$ & $<\mathrm{LOD}$ & 23.5 & 35.6 & 602.0 & $<\mathrm{LOD}$ & $<$ LOD & $<\mathrm{LOD}$ & 141.6 \\
\hline Lycicoside II & $<\mathrm{LOD}$ & $<\mathrm{LOD}$ & $<\mathrm{LOD}$ & $<\mathrm{LOD}$ & $<\mathrm{LOD}$ & $<\mathrm{LOD}$ & $<\mathrm{LOD}$ & $<\mathrm{LOD}$ & $<\mathrm{LOD}$ & 1852.0 & 72.2 \\
\hline Macranthoside A & 7.3 & $<\mathrm{LOD}$ & 24.0 & 8.6 & $<\mathrm{LOD}$ & 9.3 & 229.6 & 40.2 & 1553.2 & 63.9 & 49.2 \\
\hline Collinsonidin & 232.3 & $<\mathrm{LOD}$ & $<$ LOD & $<$ LOD & 2.4 & $<\mathrm{LOD}$ & 10.4 & 46.0 & 118.4 & $<\mathrm{LOD}$ & $<\mathrm{LOD}$ \\
\hline Dipsacus saponinA & 181.6 & 38.8 & 44.8 & 33.4 & 197.2 & 17.1 & 540.6 & 150.4 & 7999.0 & 1059.9 & 83.3 \\
\hline$\alpha$-hederin & 193.5 & 40.5 & 62.0 & 41.3 & 223.6 & 22.1 & 685.6 & 144.3 & 8734.2 & 1079.9 & 107.0 \\
\hline Pomolic acid & $<\mathrm{LOD}$ & 9878.82 & 5137.0 & 1998.8 & $<\mathrm{LOD}$ & 5166.0 & 11166.6 & 32076.4 & $<\mathrm{LOD}$ & 3997.9 & $<\mathrm{LOD}$ \\
\hline
\end{tabular}


Saponins from Cephalaria species

Table 2 continued.

\begin{tabular}{|c|c|c|c|c|c|c|c|c|c|c|c|}
\hline \multirow[t]{2}{*}{ Compounds } & \multicolumn{11}{|c|}{ Cephalaria Species } \\
\hline & 12 & 13 & 14 & 15 & 16 & 17 & 18 & 19 & 20 & 21 & 22 \\
\hline Gazipashoside B & $<\mathrm{LOD}$ & $<\mathrm{LOD}$ & $<\mathrm{LOD}$ & $<\mathrm{LOD}$ & $<\mathrm{LOD}$ & $<\mathrm{LOD}$ & $<\mathrm{LOD}$ & $<\mathrm{LOD}$ & $<\mathrm{LOD}$ & $<\mathrm{LOD}$ & $<\mathrm{LOD}$ \\
\hline Aristatoside A & 57.5 & $<\mathrm{LOD}$ & $<\mathrm{LOD}$ & 22.1 & $<\mathrm{LOD}$ & 120.0 & $<\mathrm{LOD}$ & $<\mathrm{LOD}$ & $<\mathrm{LOD}$ & 34.0 & 423.0 \\
\hline Cephoside B & 33.9 & 10.62 & 72.1 & 674.2 & $<\mathrm{LOD}$ & $<\mathrm{LOD}$ & 6.9 & 5.5 & 80.7 & 115.0 & 1596.0 \\
\hline Davisianoside A & 45.8 & $<\mathrm{LOD}$ & $<\mathrm{LOD}$ & 1548.0 & $<\mathrm{LOD}$ & 38.8 & 6.4 & 2.5 & 73.6 & 74.4 & $<\mathrm{LOD}$ \\
\hline Elmalienoside $\mathrm{C}$ & 23.7 & $<\mathrm{LOD}$ & 19.3 & 34.7 & $<\mathrm{LOD}$ & 9.4 & 2.2 & 1.1 & 47.1 & 422.3 & 599.0 \\
\hline Balansoid C & $<\mathrm{LOD}$ & $<\mathrm{LOD}$ & 35.5 & 142.8 & $<\mathrm{LOD}$ & $<\mathrm{LOD}$ & $<\mathrm{LOD}$ & $<\mathrm{LOD}$ & $<\mathrm{LOD}$ & $<\mathrm{LOD}$ & 3863.0 \\
\hline Dipsacoside B & 258.6 & $<\mathrm{LOD}$ & $<\mathrm{LOD}$ & 2201.9 & $<\mathrm{LOD}$ & 152.4 & 1124.2 & 16.7 & 453.6 & 2965.2 & 1088.0 \\
\hline Elmalienoside A & 14.2 & 0.3 & 6.4 & 5176.4 & $<\mathrm{LOD}$ & $<\mathrm{LOD}$ & 16.6 & $<\mathrm{LOD}$ & $<\mathrm{LOD}$ & 213.9 & 65.0 \\
\hline TchihatchewosideA & 48.6 & 10.9 & 5773.4 & 1216.5 & 389.2 & 78.2 & 108.3 & 21.9 & 177.1 & $<\mathrm{LOD}$ & 308.2 \\
\hline Scoposide B & 412.5 & $<\mathrm{LOD}$ & $<\mathrm{LOD}$ & 0.9 & $<\mathrm{LOD}$ & 8.8 & 0.5 & 0.6 & 152.9 & 11.7 & $<\mathrm{LOD}$ \\
\hline TchihatchewosideB & 39.8 & $<\mathrm{LOD}$ & $<\mathrm{LOD}$ & 18.6 & $<\mathrm{LOD}$ & 48.8 & 25.3 & 11.4 & 100.0 & 2304.4 & 98.0 \\
\hline Saponin I & 44.7 & 3.2 & $<\mathrm{LOD}$ & 82.6 & $<\mathrm{LOD}$ & 31.8 & 13.9 & 5.2 & $<\mathrm{LOD}$ & 222.9 & 129.0 \\
\hline Balansoid B & 572.3 & $<\mathrm{LOD}$ & $<\mathrm{LOD}$ & $<\mathrm{LOD}$ & $<\mathrm{LOD}$ & 40.0 & 5.5 & 2.9 & 1143.6 & 105.3 & $<\mathrm{LOD}$ \\
\hline Aristatoside C & 19.7 & 7.8 & $<\mathrm{LOD}$ & 9.5 & $<\mathrm{LOD}$ & $<\mathrm{LOD}$ & $<\mathrm{LOD}$ & 6.9 & 103.6 & 162.2 & $<\mathrm{LOD}$ \\
\hline Isacoside & $<\mathrm{LOD}$ & $<\mathrm{LOD}$ & $<\mathrm{LOD}$ & $<\mathrm{LOD}$ & $<\mathrm{LOD}$ & $<\mathrm{LOD}$ & $<\mathrm{LOD}$ & $<\mathrm{LOD}$ & $<\mathrm{LOD}$ & $<\mathrm{LOD}$ & $<\mathrm{LOD}$ \\
\hline Akebia Saponin F & 280.6 & $<\mathrm{LOD}$ & $<\mathrm{LOD}$ & 82.3 & $<\mathrm{LOD}$ & 38.8 & $<\mathrm{LOD}$ & 7.2 & 877.1 & 402.5 & 78.1 \\
\hline Sapindoside C & 58.0 & 25.7 & $<\mathrm{LOD}$ & $<\mathrm{LOD}$ & $<\mathrm{LOD}$ & $<\mathrm{LOD}$ & $<\mathrm{LOD}$ & 18.7 & 291.4 & $<\mathrm{LOD}$ & $<\mathrm{LOD}$ \\
\hline Tormentic acid & 3233.9 & 2257.6 & 27781.1 & 1283.2 & 6484.9 & 15851.8 & 74.0 & 805.0 & 16598.6 & 3393.3 & 75702.3 \\
\hline Balansoid A & $<\mathrm{LOD}$ & $<\mathrm{LOD}$ & 32.1 & 13.2 & $<\mathrm{LOD}$ & $<\mathrm{LOD}$ & 4.1 & $<\mathrm{LOD}$ & $<\mathrm{LOD}$ & $<\mathrm{LOD}$ & 322.4 \\
\hline Davisianoside B & $<\mathrm{LOD}$ & $<\mathrm{LOD}$ & $<\mathrm{LOD}$ & 284.8 & $<\mathrm{LOD}$ & $<\mathrm{LOD}$ & $<\mathrm{LOD}$ & $<\mathrm{LOD}$ & $<\mathrm{LOD}$ & $<\mathrm{LOD}$ & $<\mathrm{LOD}$ \\
\hline Lycicoside II & $<\mathrm{LOD}$ & $<\mathrm{LOD}$ & $<\mathrm{LOD}$ & $<\mathrm{LOD}$ & $<\mathrm{LOD}$ & $<\mathrm{LOD}$ & $<\mathrm{LOD}$ & $<\mathrm{LOD}$ & $<\mathrm{LOD}$ & $<\mathrm{LOD}$ & $<\mathrm{LOD}$ \\
\hline Macranthoside A & $<\mathrm{LOD}$ & 6.3 & $<\mathrm{LOD}$ & 515.8 & $<\mathrm{LOD}$ & $<\mathrm{LOD}$ & $<\mathrm{LOD}$ & 6.0 & $<\mathrm{LOD}$ & 67.6 & 195.00 \\
\hline Collinsonidin & $<\mathrm{LOD}$ & $<\mathrm{LOD}$ & 145.2 & $<\mathrm{LOD}$ & 16.8 & $<\mathrm{LOD}$ & $<\mathrm{LOD}$ & 32.6 & $<\mathrm{LOD}$ & $<\mathrm{LOD}$ & $<\mathrm{LOD}$ \\
\hline Dipsacus saponinA & 51.5 & 17.4 & 59.5 & 149.8 & 38.3 & 130.0 & 401.5 & 45.4 & 239.3 & 514.5 & 500.8 \\
\hline$\alpha$-hederin & 53.5 & 18.6 & $<\mathrm{LOD}$ & 172.6 & $<\mathrm{LOD}$ & 166.5 & 418.0 & 49.4 & 272.1 & 580.8 & 539.1 \\
\hline Pomolic acid & 4482.5 & 4636.1 & 113426.6 & 5403.4 & 10987.2 & 20823.5 & $<\mathrm{LOD}$ & $<\mathrm{LOD}$ & 38892.9 & $<\mathrm{LOD}$ & 180542.8 \\
\hline
\end{tabular}


Boke Sarikahya et.al., Rec.Nat.Prod. 15:6 (2021) 537-546

According to the results of LC-HRMS analysis; while the major saponins were found as dipsacus saponin A [40] and $\alpha$-hederin [41], major triterpene compounds were detected as tormentic and pomolic acids [50] in the $n$-butanol extracts of Cephalaria species. The main triterpene compound, tormentic acid was presented at the level between $74.0 \mathrm{mg} / \mathrm{kg}$ and $75702.3 \mathrm{mg} / \mathrm{kg}$ in $n$-butanol extract of all Cephalaria species. The other triterpene pomolic acid was also detected in remarkable amounts in eight Cephalaria species such as $180.5 \mathrm{~g} / \mathrm{kg}$ in C. cilicica.

The saponins $\alpha$-hederin and dipsacus saponin A were detected at the level between 22.1-8734.2 $\mathrm{mg} / \mathrm{kg}$ and $17.1-7999.0 \mathrm{mg} / \mathrm{kg}$, respectively. Balansoide $\mathrm{C}$, which was determined as the highest saponin, was found as $14776.4 \mathrm{mg} / \mathrm{kg}$ in C. balansae.

This is the first report to prove that Cephalaria species can be a good source of tormentic acid. In our previous study, where we focused on the apolar fraction of $C$. tuteliana species, we isolated and structurally determined tormentic acid from this plant [43]. Tormentic acid has many biological activities such as anti-inflammatory [51], antibacterial, anti-biofilm [52], anti-allodynia [22], immunomodulatory [53], anti-tumor, anti-atherogenic activities [54] and be used not only in pharmaceutical areas but also in cosmetic industry. According to many literature and patents; tormentic acid or its derivatives have potential interest in the development of new clinically relevant drugs for the management of proliferative vascular diseases, diabetes, osteoarthritis, hyperlipidemia and skin disorders [22, 52-54].

In our previous biological activity study [16], aristatoside C and davisianoside B which are the monodesmosidic saponins with hederagenin aglycone displayed significant inhibitory effects on cancerous A549 and HeLa cells with $\mathrm{IC}_{50}$ values of $3.52 \pm 0.11,35.69 \pm 0.50 \mu \mathrm{M}$ and $4.08 \pm 0.06,11.74$ $\pm 0.82 \mu \mathrm{M}$, respectively. They are the cytotoxic compounds against lung cancer cells with greater activity than standard commercial chemotherapy drug doxorubicin and davisianoside B also displayed less toxic effect than doxorubicin against non-cancerous kidney cell line HEK-293 [16]. Aristatoside C was found in thirteen Cephalaria species (C. tchihatchewii, C. scoparia, C. isaurica, C. taurica, C. aytachii, C. aristata, C. paphlagonica, C. lycica, C. elazigensis var. purpurea, C. anatolica, C. procera, C. gazipashaensis and C. hirsuta) presented in good amounts at the level between 3.4 to $7030.8 \mathrm{mg} / \mathrm{kg}$. Besides that, davisianoside B was detected in five Cephalaria species (C. sumbuliana, C. taurica, C. aytachii, C. paphlagonica and C. anatolica) at the level between 23.5 and $602.0 \mathrm{mg} / \mathrm{kg}$.

Another finding from the same biological activity study is that some saponins such as elmalienoside A, balansoide B, scoposide B induced remarkable IL- $1 \beta$ and IFN- $\gamma$ secretions $[15,16]$. So, it is possible for these saponins to be evaluated as vaccine adjuvant candidates for different types of cancer. Therefore, it is very important to identify these saponins in different Cephalaria species. While elmalienoside A was detected in all Cephalaria species except $C$. stellipilis, $C$. procera, $C$. gazipashaensis, C. elmaliensis from $1.8 \mathrm{mg} / \mathrm{kg}$ to $5176.4 \mathrm{mg} / \mathrm{kg}$, balansoide B was found in good amounts between $1.9 \mathrm{mg} / \mathrm{kg}$ and $1143.6 \mathrm{mg} / \mathrm{kg}$ in all Cephalaria species except $C$. davisiana, C. aristata, $C$. elazigensis var. purpurea, C. elazigensis var. elazigensis, C. anatolica C. elmaliensis, C. cilicica. In addition, scoposide B which is a minor saponin with oleanoic acid aglycone was established in twelve Cephalaria species (C. tchihatchewii, C. scoparia, C. sumbuliana, C. davisiana, C. paphlagonica, $C$. lycica, C. anatolica, C. stellipilis, C. speciosa, C. procera, C. gazipashaensis and C. hirsuta) at the level between 0.4 and $412.5 \mathrm{mg} / \mathrm{kg}$.

\section{Conclusion}

In the last decade, saponins isolated from a number of plants have demonstrated a wide range of biological and pharmacological activities in many in vitro and in vivo bioassays. In this study, we provided a broad screening of important biologically active triterpenes and saponins by LC-HR/MS method. In summary; this method with high separation ability, high sensitivity and high selectivity has been successfully established for the simultaneous determination of twenty-six biologically active saponins and triterpenes in 22 Cephalaria species, for the first time. Therefore, due to the different activities and usages of the identified compounds, these findings may be useful for a number of industries such as nutraceutical, pharmaceutical, medical and cosmetic industries. 


\section{Conflicts of Interest}

The authors have declared that there are no conflicts of interest.

\section{Acknowledgements}

The authors gratefully thank Prof. Dr. H. Sumbul and Prof. Dr. R. S. Gokturk for the collection and identification of the plant materials.

\section{Supporting Information}

Supporting information accompanies this paper on http://www.acgpubs.org/journal/records-ofnatural-products

\section{ORCID}

Nazli Boke Sarikahya: 0000-0002-8200-8561

Ahmet C Goren: 0000-0002-5470-130X

Gaye Sumer Okkali: 0000-0003-2756-0617

Suheyla Kirmizigul: $\underline{0000-0001-8514-4123}$

\section{References}

[1] M. Guclu-Ustundag and G. Mazza (2007). Saponins: properties, applications and processing, Crit. Rev. Food Sci. Nutr. 47, 231-258.

[2] I. Podolak, A. Galanty and D. Sobolewska (2010). Saponins as cytotoxic agents: a review, Phytochem Rev. 9, 425-474.

[3] S.G. Sparg, M.E. Light and J. Van Staden (2004). Biological activities and distribution of plant saponins, $J$. Ethnopharmacol. 94, 219-243.

[4] M.A. Lacaille-Dubois and H. Wagner (1996). A review of the biological and pharmacological activities of saponins, Phytomedicine 2, 363-386.

[5] A. Nalbantsoy, T. Nesil, S. Erden, I. Calis and E. Bedir (2011). Adjuvant effects of Astragalus saponins macrophyllosaponin B and astragaloside VII, J. Ethnopharmacol. 134, 897-903.

[6] G. Ragupathi, J.R. Gardner, P.O. Livingston and D.Y. Gin (2011). Natural and synthetic saponin adjuvant QS-21 for vaccines against cancer, Expert Rev. Vaccines 10(4), 463-470.

[7] N. Yakubogullari, R. Genc, F. Coven, A. Nalbantsoy and E. Bedir (2019). Development of adjuvant nanocarrier systems for seasonal influenza A (H3N2) vaccine based on Astragaloside VII and gum tragacanth, Vaccine 37(28), 3638-3645.

[8] N.B. Sarikahya and S. Kirmizigul (2010). Antimicrobial triterpenoid glycosides from Cephalaria scoparia, J. Nat. Prod. 73, 825-830.

[9] K. Mustafayeva, C.D. Giorgio, R. Elias, Y. Kerimov, E. Ollivier and M. De Meo (2010). DNA-Damaging, mutagenic, and clastogenic activities of gentiopicroside isolated from Cephalaria kotschyi roots, J. Nat. Prod. 73, 99-103.

[10] N. Tabatadze, R. Elias, R. Faure, P. Gerkens, M.C. De Pauw-Gillet, E. Kemertelidze, A. Chea and E. Ollivier (2007). Cytotoxic triterpenoid saponins from the roots of Cephalaria gigantea, Chem. Pharm. Bull. 55, 102105.

[11] S. Pasi, N. Aligiannis, H. Pratsinis, A.L. Skaltsounis and I.B. Chinou (2009). Biologically active triterpenoids from Cephalaria ambrosioide, Planta Med. 75, 163-167.

[12] D. Godjevac, V. Vajs, N. Menkovic, V. Tesevic, P. Janackovic and S. Milosavljevic (2004). Flavonoids from flowers of Cephalaria pastricensis and their antiradical activity, J. Serbian Chem. Soc. 69, 883-886.

[13] P.H. Davis. (1972). Flora of Turkey and the east Aegean Islands, Edinburgh: University of Edinburgh Press; 4, 585-592.

[14] R.S. Gokturk and H. Sumbul (2014). A taxonomic revision of the genus Cephalaria (Caprifoliaceae) in Turkey, Turk. J. Botan. 38, 927-968.

[15] H. Top, N.B. Sarikahya, A. Nalbantsoy and S. Kirmizigul (2017). Immunomodulatory, hemolytic properties and cytotoxic activity potent of triterpenoid saponins from Cephalaria balansae, Phytochemistry 137, 139147. 
Boke Sarikahya et.al., Rec.Nat.Prod. 15:6 (2021) 537-546

[16] N.B. Sarikahya, A. Nalbantsoy, H. Top, R.S. Gokturk, H. Sumbul and S. Kirmizigul (2018). Immunomodulatory, hemolytic and cytotoxic activity potentials of triterpenoid compounds from eight Cephalaria species, Phytomedicine 38, 35-144.

[17] N.B. Sarikahya (2014). Aristatosides A-C, hederagenin-type triterpene saponins from Cephalaria aristata, Phytochem. Lett. 8, 149-155.

[18] N.B. Sarikahya and S. Kirmizigul (2012). Antimicrobially active hederagenin glycosides from Cephalaria elmaliensis, Planta Med. 78, 828-833.

[19] N.B. Sarikahya and S. Kirmizigul (2012). Novel biologically active glycosides from the aerial parts of Cephalaria gazipashensis, Turk. J. Chem. 36, 323-334.

[20] S. Kirmizigul, H. Anil, F. Ucar and K. Akdemir (1996). Antimicrobial and antifungal activities of three new triterpenoid glycosides, Phytother. Res. 10, 274-276.

[21] S. Kirmizigul, H. Anil and M. E. Rose (1995). Triterpenoid glycosides from Cephalaria transsylvanica, Phytochemistry 39, 1171-1174.

[22] B.B. Leslei, F. Juliano, C.H. Sonia, D.M. Franco, A.Y. Rosendo and B.C. Joao (2002). Anti-allodynic action of the tormentic acid, a triterpene isolated from plant, against neuropathic and inflammatory persistent pain in mice, Eur. J. Pharmacol. 453(2-3), 203-208.

[23] J. Sun, Y. Feng, Y. Wang, Q. Ji, G. Cai, L. Shi, Y. Wang, Y. Huang, J. Zhang and Q. Li (2019). $\alpha$-Hederin induces autophagic cell death in colorectal cancer cells through reactive oxygen species dependent AMPK/mTOR signaling pathway activation, Int. J. Oncol. 54, 1601-1612.

[24] Y-M. Zhao and Y-P. Shi (2011). Phytochemicals and biological activities of Dipsacus species, Chem. Biodivers. 8, 414-430.

[25] N.B. Sarikahya, A.C. Goren and S. Kirmizigul (2019). Simultaneous determination of several flavonoids and phenolic compounds in nineteen different Cephalaria species by HPLC-MS/MS, J. Pharm. Biomed. Anal. $173,120-125$.

[26] G. Sumer, N.B. Sarikahya and S. Kirmizigul (2017). Phytochemical and biological investigations on Cephalaria anatolica, Rec. Nat. Prod. 11(6), 497-507.

[27] P. Kayce, N.B. Sarikahya and S. Kirmizigul (2014). Two novel saponins from Cephalaria davisiana (Dipsacaceae), Phytochem. Lett. 10, 324-329.

[28] M.M. Mukhamedziev, P.K. Alimbaeva, T.T. Gorovits and N.K. Abubakirov (1971). Structure of the triterpenoid glycoside from Dipsacus azureus, Dipsacoside B, Khim. Prir. Soedin. 7, 153-158.

[29] N.B. Sarikahya, M.C. Mkouboi, A. Nalbantsoy and M. Elibol (2021). Cytotoxic and immunomodulator potential of hederagenin saponins from Cephalaria tchihatchewii, Phytochem. Lett. (in decision).

[30] N.B. Sarikahya, M. Pekmez, N. Arda, P. Kayce, N.U. Karabay Yavasoglu and S. Kirmizigul (2011). Isolation and characterization of biologically active glycosides from endemic Cephalaria species in Anatolia, Phytochem. Lett. 4(4), 415-420.

[31] H. Kawai, M. Kuroyanagi, K. Umehara, A. Ueno and M. Satake (1988). Studies on the saponins of Lonicera japonica Thunb, Chem. Pharm. Bull. 36, 4769-4775.

[32] A. Braca, G. Autore, F. De Simone, S. Marzocco, I. Morelli, F. Venturella and N. De Tommasi (2004). Cytotoxic saponins from Schefflera rotundifolia, Planta Med. 70, 960-966.

[33] H. Abaci, G. Akagac, A. Nalbantsoy and N.B. Sarikahya (2021). A hederagenin-type triterpene saponin, sumbulianoside A from Cephalaria sumbuliana and its potent immunomodulatory activity against seasonal flu virus H3N2, Nat. Prod. Res. 1-8. doi: 10.1080/14786419.2021.1910691.

[34] P. Kayce and S. Kirmizigul (2010). Chemical constituents of two endemic Cephalaria species, Rec. Nat. Prod. 4, 141-148.

[35] R. Higuchi and T. Kawasaki (1972). Seed saponins of Akebia quinata DECNE. II. Hederagenin 3, 28-Obisglycosides, Chem. Pharm. Bull. 20, 2143-2149.

[36] V.Y. Chirva, P.K. Kintya and V.A. Sosnovskii (1970). Triterpenic glycosides of Sapindus mukorossi. III. Structure of sapindoside C, Khim. Prir. Soedin. 6(3), 374-375.

[37] E. Halay and S. Kirmizigul (2010). Glycosides from Cephalaria species, Z. Naturforsch B. 65, 13841392.

[38] S. Saito, S. Sumita, N. Tamura, Y. Nagamura, K. Nishida, M. Ito and I. Ishiguro (1990). Saponins from the leaves of Aralia elata seem (Araliaceae), Chem. Pharm. Bull. 38, 411-414.

[39] B.S. Joshi, K.M. Moore, S.W. Pelletier, M.S. Puar and B.N. Pramanik (1992). Saponins from Collinsonia candensis, J. Nat. Prod. 55, 1468-1476.

[40] K.Y. Jung, K.H. Son and J.C. Do (1993). Triterpenoids from the roots of Dipsacus asper, Arch Pharm. Res. 16(1), 32-35.

[41] A.M. Aliev and I.S. Movsumov (1976). Triterpenoid glycosides from Cephalaria kotchyi flowers, Khim. Prir. Soedin. 2, 264-265. 
[42] P. Kayce, N.B. Sarikahya, M. Pekmez, N. Arda and S. Kirmizigul (2017). The structure and cytotoxic activity of a new saponin: Cephoside A from Cephalaria elazigensis var. purpurea, Turk. J. Chem. 41(3), 345-353.

[43] M. Dagli, N.B. Sarikahya, A. Nalbantsoy and S. Kirmizigul (2019). Comparative phytochemical screening and cytotoxic efficacy of endemic Cephalaria tuteliana, Nat. Prod. Commun. 14, 1-5.

[44] H.O. Hamad, M.H. Alma, I. Gulcin, M.A. Yilmaz and E. Karaogul (2017). Evaluation of phenolic contents and bioactivity of root and nutgall extracts from iraqian Quercus infectoria olivier, Rec. Nat. Prod. 11, 205210.

[45] A.C. Goren, G. Bilsel and M. Bilsel (2007). Rapid and simultaneous determination of 25-OH-vitamin D 2 and D 3 in human serum by LC/MS/MS: Validation and uncertainty assessment, J. Chem. Metrol. 1, 1-9.

[46] F. Akbas, A. Ozaydin, E. Polat and I. Onaran (2020). Lucilia sericata larval secretions stimulating wound healing effects on rat dermal fibroblast cells, Rec. Nat. Prod. 14(5), 340 -354.

[47] M. Topal (2020). Secondary metabolites of ethanol extracts of Pinus sylvestris cones from Eastern Anatolia and their antioxidant, cholinesterase and $\alpha$-glucosidase activities, Rec. Nat. Prod. 14(2), 129-138.

[48] Z. Ozer, S. Carikci, H. Yilmaz, T. Kilic, T. Dirmenci and A.C. Goren (2020). Determination of secondary metabolites of Origanum vulgare subsp. hirtum and $O$. vulgare subsp. vulgare by LC-MS/MS, J. Chem. Metrol. 14, 25-34.

[49] D. Dincel, H. Olgan, Z. Canbaloğlu, S. Yalcin, A. Erkuçuk, G. Tırıs and A.C. Gören (2020). Determination of dihydrocapsaicin adulteration in dietary supplements using LC-MS/MS, J. Chem. Metrol. 14, 77-82.

[50] A. Villar, M. Paya, M.D. Hortiguela and D. Cortes (1986). Tormentic acid, a new hypoglycemic agent from Poterium ancistroides, Planta Med. 52, 43-45.

[51] M. Chikako, K. Ishijima, M. Hirota, K. Sakaguchi, H. Yoshida and Y. Mizushina (2002). Novel antiinflammatory compounds from Rubus sieboldii, triterpenoids, are inhibitors of mammalian DNA polymerases, Biochim. Biophys. Acta. 1596(2), 193-200.

[52] C. Tafadzwa, B. Genuine, M. Tatenda, S. Simbarashe, M. Stanley and F. C. Godloves (2020). An evaluation of the antibacterial properties of tormentic acid congener and extracts from Callistemon viminalis on selected ESKAPE pathogens and effects on biofilm formation, Adv. Pharmacol. Pharm. Sci. 2020, 8848606.

[53] Y. Yang, J. Haobo, L. Bing, X. Dan, W. Yawei and Z. Meng (2018). Tormentic acid inhibits IL-1 $\beta$-induced chondrocyte apoptosis by activating the PI3K/Akt signaling pathway, Mol. Med. Rep. 17(3), 4753-4758.

[54] S.F. Anelize, A. Eliane, B.C. Joao and H.C. Alexandre (2009). Tormentic acid reduces vascular smooth muscle cell proliferation and survival, Eur. J. Pharmacol. 615(1-3), 50-54.

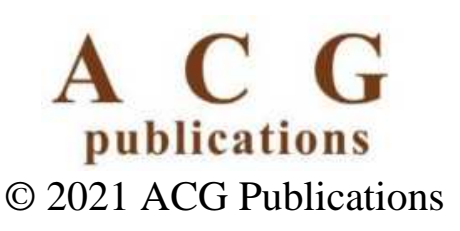

